\title{
Sistemas de vida complejos como vía de aproximación analítica a los pueblos originarios
}

\author{
Complex life systems as a way of analytical approach to native peoples
}

Ignacio Ramos Beltrán ${ }^{a}$

\begin{abstract}
:
The process of approaching and understanding ancestral cultures, as well as the theoretical-methodological tools used, has generated a great deal of reflection and restructuring in the same conceptions and strategies used in the work carried out in this field. This reflective and analytical process has gone through several stages. What is shown in this article is a restructuring of the theoreticalmethodological scaffolds from which it is possible to visualize and carry out the work in the original communities or ancestral peoples. The proposal is based on a systemic approach. It must be taken into account that concepts or terms such as: complexity, systems, transdiscipline, networks, self-organization, structure, etc., have been transformed over time. Subjects and cultures live and update inherited knowledge and practices, which when transmitted also undergo transformations. All this makes up complex swarms and knots of knowledge and practices. Considering cultures as non-improvised forms of life allows us to have a broader and deeper understanding of the phenomena we are approaching. Therefore, sharing these experiences is essential, considering the complexity of the plots, epistemic assumptions and universes of discourse that surround them.
\end{abstract}

Keywords:

Culture, Systems of Life, Transdiscipline, Self-organization, Complexity

Resumen:

El proceso de acercamiento y entendimiento a las culturas ancestrales, así como las herramientas teórico-metodológicas empleadas, ha generado una gran reflexión y reestructuración en las mismas concepciones y estrategias empleadas en los trabajos realizados en este campo. Este proceso reflexivo y analítico ha atravesado varias etapas. Lo que se muestra en este artículo es una reestructuración de los andamios teórico-metodológicos desde los cuales es posible visualizar y realizar el trabajo en las comunidades originarias o pueblos ancestrales. La propuesta tiene como base una aproximación sistémica. Es preciso tener en cuenta que las concepciones o términos tales como: complejidad, sistemas, transdisciplina, redes, auto-organización, estructura, etcétera, se han ido transformando con el tiempo. Los sujetos y las culturas viven y actualizan saberes y prácticas heredadas, las cuales al ser transmitidas también sufren transformaciones. Todo ello conforma enjambres y nudos complejos de saberes y de prácticas. Considerar a las culturas como formas de vida no improvisadas, nos permite tener una comprensión más amplia y profunda de los fenómenos a los que nos acercamos. Por tanto, compartir dichas experiencias es fundamental, considerando la complejidad de las tramas, supuestos epistémicos y universos de discurso que las rodean.

Palabras Clave:

Cultura, Sistemas de Vida, transdisciplina, Auto-organización, Complejidad

\section{Introducción}

Las culturas entendidas como complejos vivos de saberes nos muestran matices diversos de formas de vidas heredadas, compartidas y transmitidas. Los mecanismos y los contextos, los procesos históricos y los procesos emergentes son diversos; de ahí la importancia de observar la complejidad de cada ensayo o puesta en escena que realizan las comunidades a las que se les ha llegado a denominar pueblos originarios o culturas ancestrales. La pobreza 0 artificialidad al intentar entendernos a nosotros, y al intentar entender a otros pueblos, ha provocado que en muchos casos se lleguen a realizar sobreactuaciones intelectuales o eruditas, las cuales sólo generaran algunos espasmos de originalidad

\footnotetext{
a Autor de Correspondencia, Universidad Nacional Autónoma de México, ORCID: http://orcid.org/0000-0002-6024-4766,
}

Email: ignamesoamerica@yahoo.com.mx 
o rebuscados ejercicios conceptuales, que pocas veces son aplicables o utilizados por las comunidades mismas. La concepción de lugar y de tiempo que viven y habitan los pobladores de estos distintos entornos culturales, es un proceso que se crea y recrea constantemente. Tómese como un ejemplo el caso de los museos comunitarios.

Dichos espacios, en muchas ocasiones, se convierten en vestimentas sociales, las cuales pueden ser una máscara "activa" o "contemplativa" de estas comunidades, como lo refieren Questa y Neurath, los que tienen el "inefable atractivo de ver un rostro extraño y de poder portarlo temporalmente, [...] como un artefacto común" (Questa y Neurath, 2018:10).

Esta puede ser la puerta de entrada que nos permita vislumbrar la propuesta que tenemos en mente. Desde una perspectiva más amplia, compleja y sistémica, lo que tendríamos que tratar de entender son los juegos de los distintos sujetos sociales implicados en los tejidos sociales que se producen, y observar el sentido de la trama que recorren. Esto es, todos los hilos forman parte de un tejido que es preciso descifrar: la comunidad, las costumbres, las formas de divertirse, la comida, la distribución de los espacios, los templos, los museos, etc. En otras palabras, en todo sistema social vivo, la máscara que se nos muestra o se nos presenta forma parte de formas complejas de convivencia y respeto.

Las relaciones en las que se han entrelazado diferentes hilos, por parte de los sujetos culturales, muestran redes entremezcladas de certezas e incertidumbres, miedos y alegrías, así como la posibilidad de observar infinitos y nuevos caminos de la cotidianidad. La inmensidad de ángulos y formas de vida de los sujetos o actores que nos salen al encuentro es innumerable. Las redes que tejen entre todos ellos toman forma a través de una concepción de tiempo que juega a ser varios tiempos a la vez. Una cosa semejante sucede con los espacios, los paisajes se transforman.

Como bien mencionan los autores de 'Las Vías del Noreste', al hablar de problemáticas culturales hay que hacer alusión a macro-regiones y/o corredores culturales, lo cual nos hace reparar en la necesidad de distinguir conjuntos sistémicos de ideas y de prácticas con las cuales dialogan los diferentes pueblos. Es necesario entonces, hacer énfasis y un señalamiento en relación a este aspecto: la complejidad de los fenómenos no sólo se encuentra en el campo mismo, esto es, en las comunidades, sino también en un grupo de conceptos teórico-conceptuales, con los cuales nos acercamos a esos fenómenos o eventos. Hablar de macro-regiones o de corredores culturales no es un elemento que salte a primera vista o de inmediato cuando uno se encuentra en un poblado. Es común quedar atrapado por ese mismo poblado, en el que uno se encuentra o trabaja, en ese momento; ignorando o pensando que los pueblos aledaños no existen. Por ejemplo, en el caso concreto de los rituales dancísticos, como ‘el bailar fariseo' o el 'bailar matachín', para lograr "la comprensión de la variante tarahumara, sólo puede llevarse a cabo con plenitud si esta danza es analizada dentro del macrosistema cultural -taracahíta, corachol y pueblo- del que forma parte" (Bonfiglioli, Gutiérrez, \& Olavarría, 2006:23). En el caso de las investigaciones realizadas en el Gran Nayar, los autores señalan lo siguiente:

Se puede señalar que las religiones indígenas contemporáneas del noroeste abrevan de un conjunto de corrientes que confluyen en una tradición más amplia, como la de pueblo o la mesoamericana; por lo que un mayor acercamiento al fenómeno del "sincretismo" (Báez, 1998) revelará que no basta entenderlo como una apropiación de elementos readecuados a la situación de contacto cultural o como mecanismo estabilizador, sino como un mecanismo creativo que refuncionaliza los elementos sobre bases estructurales comunes

(Bonfiglioli, Gutiérrez, \& Olavarría, 2006:24).

Desde la semiótica de la cultura, como lo muestra Lotman, la cultura se entiende como un sistema semiótico generador de otros sistemas, en donde la relación dinámica y estática de éste conduce al planteamiento de una pregunta clave: “¿de qué manera un sistema puede desarrollarse permaneciendo él mismo?", esto es, “¿cómo se mantiene la relación entre sistema, realidad externa, y su recíproca impenetrabilidad y/o permeabilidad?" (Lotman, 1999:11).

Aquí comenzamos a cabalgar sobre uno de los conceptos centrales de la propuesta a considerar en este artículo: el término sistema. Como se señala en el prólogo del libro titulado, 'La población hablante de lenguas indígenas en México':

En la actualidad, en pleno siglo XXI, los indígenas aún son sometidos al racismo, la discriminación y la marginación. Para coadyuvar en el conocimiento de esta injusticia, y en aras de atender los rezagos existentes, un paso necesario es valorar, entre otros aspectos, la importancia de las lenguas indígenas como herramientas para facilitar la educación básica de estos grupos... (Ortiz, 2005: 10).

Si atendemos a la concepción de sistema que propone Lotman, es posible observar que la relación dinámica del sistema nos obliga a considerar y tomar en cuenta varios elementos a la vez: lengua, rezago cultural, educación, alimentación, vivienda, entre otros. Gran cantidad de las investigaciones, realizadas en los distintos centros o institutos en México sobre poblaciones indígenas, son excelentes trabajos y proveen una infinita gama de datos e información que pueden ayudar a realizar propuestas o acercamientos mucho más acabados y reflexivos, 
dependiendo de la dificultad de la problemática tratada, sin embargo no son suficientes.

Aunque parezca redundante, y lo volveremos a recordar una y otra vez, en el caso de los pueblos originarios o culturas ancestrales en México, se ha observado la emergencia de complejas tramas de relaciones en una reformulación constante tanto al interior como al exterior de las culturas mismas, y por ende, también de los sujetos que las conforman: salud, legalidad, lengua, tierra, conciencia, agua, naturaleza, vida, etcétera. Por lo que en este documento pretendemos mostrar, a través de un conjunto de conceptos trabajados y reelaborados desde las teorías de la complejidad y de los sistemas, que las comunidades indígenas son sistemas que se crean y se recrean a sí mismos, al transformar sus propios componentes, lo cual implica que sufren cambios estructurales continuos, y que al mismo tiempo, preservan un patrón-red de organización, lo cual indica que la forma y extensión de la organización humana está viva.

Desde la concepción de 'los sistemas de vida', desarrollada por Fritjob Capra y Pier Luigi Luisi, es posible observar que lo realizado por distintas comunidades o pueblos originarios, puede ser entendido como una dinámica propia de los sistemas abiertos de autogenerar redes que reemplazan y/o transforman otros componentes del sistema mismo (Capra \& Luisi, 2018:105). Las dinámicas de estos sistemas abiertos no lineales (como son las comunidades de los pueblos indígenas) es la de hacer que emerjan nuevos órdenes, dentro de los mismos sistemas, en los puntos críticos de la inestabilidad del mismo. La importancia y pertinencia de esta propuesta consiste en darnos cuenta que la concepción de 'sistemas de vida' es el resultado del trabajo con fenómenos naturales de diferente orden de complejidad: desde trabajos de laboratorio en disciplinas como la física o la biología, hasta los eventos sociales en donde la cultura es entendida también como sistema.

En México se reporta la existencia de 68 lenguas indígenas, además del español, que el Instituto Nacional de Lenguas Indígenas (INALI, 2020) cristaliza en una serie animada, con el título, "68 voces - 68 corazones", con la que se evidencia la amplitud del conjunto de universos que se han perdido, muchas veces de manera insospechada en el país. Estas comunidades se han organizado en diferentes formas y aspectos, y de alguna manera, han estado propiciando un diálogo, al cual no se ha sabido responder adecuadamente: el diálogo con los otros, la otredad de la que ahora tanto se habla. La variedad y diversidad de cambios y trasformaciones de patrones de organización y de redes estructurales de muchos grupos sociales, es posible observarla en procesos de desplazamiento, migración, asentamiento, búsqueda de mejores condiciones de vida, cuidado de la tierra, revitalización de la lengua, salud y enfermedad, entre otros. Todos ellos tienden redes que se insertan no sólo dentro sino fuera de las comunidades de origen, las cuales a su vez generan repercusiones o efectos de ida y vuelta.

\section{Antecedentes}

En nuestro paulatino profundizar en el entendimiento de las culturas, hemos tenido que recurrir a planteamientos epistemológicos que nos han permitido englobar y contextualizar dichos fenómenos, como fenómenos complejos. El profesor Eduardo Menéndez, en su libro: "De sujetos, saberes y estructuras", señala que existen ideas que distorsionan, reducen o evitan hacer un acercamiento relacional de los tejidos complejos que se presentan en toda cultura. Sucede con mucha frecuencia que se confunde y no se expresa de manera patente, la historicidad y el presupuesto social desde el cual se entreteje la vida. Es preciso, dice Menéndez, "Reconocer [en primer lugar] la existencia de propuestas sobre el papel decisivo de las relaciones, redes y rituales sociales en la vida colectiva [...]; y segundo, [observar] la fuerte tendencia a no describir los procesos en términos de relaciones sociales" (Menéndez, 2009:241). En otras palabras, existe, "una falta de historicidad que no incluye la dinámica continuidad/discontinuidad temporal en la construcción, resignificación, desaparición o transformación de los rituales y relaciones sociales, dado que domina en ellos una interpretación de los mismos en términos de permanencia y desaparición más que de cambio y resignificación" (Menéndez, 2009:251). Y es que, aunque parezca evidente, se nos ha enseñado a observar procesos en las relaciones sociales, que muchas veces se nos escapa observar también su historicidad, sus transformaciones. En este sentido, a principios de 2002 Capra escribió:

La visión sistémica de la vida, tal como la esbocé en The Turning Point, en 1981, no constituía una teoría coherente de los sistemas vivos, sino más bien una nueva forma de pensar sobre la vida que incluía nuevas percepciones, un nuevo lenguaje y nuevos conceptos. [...] La nueva comprensión científica de la vida, basada en los conceptos de la dinámica no lineal, representa un verdadero punto de inflexión conceptual. Disponemos, por primera vez, de un lenguaje eficaz para describir y analizar los sistemas complejos. Conceptos como los de atractor, retraso fase, diagrama de bifurcación y fractal no existían antes del desarrollo de la dinámica no lineal. Tales conceptos nos permiten hoy formular nuevas preguntas, y nos han conducido ya a importantes ideas en múltiples campos (Capra, 2002:20).

Pablo González Casanova advirtió hace más de quince años, que el trabajo en ciencias sociales se encontraba atravesando nuevos senderos y caminos, a los cuales muchos estudiosos no han hecho eco: 
Hoy ya no podemos pensar en la naturaleza, la vida y la humanidad sin tomar en cuenta los descubrimientos que se iniciaron con la cibernética, la epistemología genética, la computación, los sistemas autorregulados, adaptativos y autopoiéticos, las ciencias de la comunicación, las de la organización, las del caos determinista, los fenómenos emergentes, los atractores y fractales. La profundidad de estos descubrimientos va más allá de sus manifestaciones científicas y técnicas. Incluye nuevas formas de pensar y actuar que comprenden a las tecnociencias y a las llamadas ciencias de la complejidad (González Casanova, 2005:11).

De la misma manera, otra autora que desarrolla su trabajo en América del Sur, Denise Najmanovich, se refiere a esto como la mutación de los fundamentos de las disciplinas:

Los desarrollos del constructivismo desde los trabajos pioneros de Piaget, hasta los desarrollos cibernéticos de segundo orden de von Foerster (1991), fueron cruciales para la difusión de un nuevo modo de concebir el saber. La propuesta de Bateson (1991) para pensar la ecología de la mente, junto con los aportes de Maturana y Varela (1990) sobre la biología del conocimiento y los desarrollos del construccionismo social de Berger y Luckmann (1985), Gergen (1991) y Barnett Pearce (1989, 1994) han sido otras de las riquísimas vías de investigación que pusieron en jaque la epistemología positivista. Finalmente, las propuestas de Morin (1994a, 1994b, 1988) respecto del pensamiento complejo aportaron aguas refrescantes a esta gran ola de pensamiento epistemológico post-positivista

(Najmanovich, 2012:16).

La invitación es, de manera concreta, a insertarnos y cabalgar sobre nuevas formas de pensar y de actuar. Esos son los hechos. El impacto de esta nueva concepción sistémica ha ido ganando terreno, detalle sobre detalle, a través del trabajo metódico y particular de distintos pensadores o investigadores que observaron la utilidad y la conveniencia de tratar de acercarse y observar, a través de nuevas formas conceptuales, los fenómenos y las problemáticas naturales y sociales, como son las ideas que derivan de la auto-organización de los sistemas abiertos. Por ello resulta fundamental entender las bases de tal construcción conceptual.

En un sistema abierto, los flujos de intercambio con el exterior pueden mantener al sistema lejos de una situación de equilibrio. Bajo ciertas condiciones, el sistema puede evolucionar pasando por procesos de sucesivas desorganizaciones y reorganizaciones con estructuras cada vez más complejas... Todo sistema abierto ( $y$ todos los sistemas naturales son abiertos) evolucionan en continua interacción con el medio externo y se auto-organiza, adoptando formas de organización con estructuras que le permiten mantenerse en un cierto equilibrio dinámico con las condiciones del contorno (García, 2006:131).

Así, arte, espiritualidad, vida cotidiana, ciencia, religión, movimientos sociales, etcétera, son sistemas abiertos. Esto es, toda práctica cultural, puede ser entendida como un sistema abierto que nos muestra universos con diferentes grados de complejidad, en donde múltiples procesos se interrelacionan y van a generar, como declara Nicolescu (1997), "la adaptación de las mentalidades a esos nuevos saberes". Esto mismo, pero con distintas palabras, lo recordaba Francisco Varela hace algunos años, al recordar:

Cada época de la historia humana produce, a través de sus prácticas sociales cotidianas y su lenguaje, una estructura imaginaria. La ciencia forma parte de estas prácticas sociales, y las ideas científicas acerca de la naturaleza constituyen apenas una dimensión de esta estructura imaginaria (Varela, 2005:11-12).

\section{Fundamentos de la Propuesta}

Capra y Luisi, en su libro The Systems View of Life: A Unifying Vision, parten de una percepción sistémica de la vida que nos conduce a pensar toda problemática que encaramos en términos de relaciones, patrones y contexto. Las implicaciones son varias y quisiéramos mostrar algunos de los detalles que surgen, y que son importantes considerar, ya que en el pensamiento sistémico, cada uno de los elementos que se mencionan, se encuentran conectados entre sí en una especie de espiral continua. Esta aproximación nos permite entonces: a) percibir el mundo como una red de patrones inseparables de relaciones, y al planeta como un sistema vivo en su totalidad, el cual se auto-regula constantemente; b) la evolución, desde esta perspectiva, se ve como una interrelación de redes de patrones en los cuales la creatividad y la emergencia de la novedad son la constante de todo proceso; y c) observar que toda organización humana, como lo es toda institución social, no es sino una comunidad de gente que se auto-organiza construyendo relaciones en redes formales e informales de comunicaciones.

La vitalidad de una organización, pensada de esta manera (su flexibilidad, su creatividad potencial, su habilidad de aprendizaje), reside en que los tejidos de estas redes, no son sino "comunidades de práctica" compartidas. Son redes vivas (y toda práctica cultural forma parte de una red viva), que responden a perturbaciones con cambios estructurales, y escoge a la vez, a cuáles cambios responder y cómo responder. De esta forma, podemos observar entonces que toda cultura produce perturbaciones significativas, las cuales hacen que se preste atención a la organización desde la cual provocan que se disparen cambios estructurales en la misma (Capra \& Luisi, 2018:297-321). Pero estos 
cambios pueden provenir desde dentro o desde fuera de la estructura misma. Son estos los fundamentos epistémicos que intentamos se vuelvan el eje central de toda propuesta que pretende dar cuenta de prácticas y formas de vida de todo grupo o 'cultura ancestral', como se denominan ahora en el ámbito académico.

Nos parece que, retomando los elementos clave del trabajo desarrollado por Capra y Luisi, el universo que se despliega ante nuestros ojos mostrará con mayor amplitud y profundidad la enorme cantidad de procesos y relaciones interculturales que se han generado en los pueblos indígenas o culturas ancestrales, tales como: salud, lengua, legalidad, tierra, memoria, cuerpo, migración, danza, rituales, organizaciones comunales, sueños, etcétera. Las distintas facetas o rostros de cada pueblo pueden entenderse entonces, como parte de las respuestas que un sistema vivo manifiesta y expresa de manera sistémica. Toda cultura, y cada sujeto que vive dentro de ella, forman parte de una totalidad que se está reorganizando constantemente, a través de sus propias "comunidades de prácticas". Un acercamiento de esta clase nos ayudará a responder a una pregunta que nos hemos planteado desde hace varios años: ¿cómo es que las comunidades indígenas han desplegado y siguen desplegando estrategias tan diversas para seguir viviendo en un mundo con sentido?

Todo sujeto que nace en el mundo se encuentra rodeado de infinidad de contextos, situaciones y procesos significantes. Hallarse inmerso dentro de estos universos nos lleva a aventurarnos en lo que Henri Tajfel (1984) llama "la cotidiana empresa de vivir", y que nos conduce a desentrañar el por qué los seres humanos viven en y dentro de complejas redes de relaciones, en donde la interacción es parte de un proceso continuo y constante. Moscovici (recordando a Russel) recuerda y resalta una pregunta ingenua pero fundamental: “ ¿cómo es posible que los seres humanos, cuyos contactos con el mundo son breves, personales y limitados, sean capaces de saber tanto?" (Moscovici, 2011: XIII). La cultura, en sus múltiples formas de presentación, desde lo prosaico hasta lo poético, debe ser entendida como una unidad compleja de formas de estar en el mundo, las cuales se entienden como realidades organizadoras recursivas complejas, que concretan sus instancias y su presencia a través de su misma actividad (Morin, 2002:85).

La recopilación de trabajos que se reunieron en el libro: "Fronteras semióticas de la emoción" (Haidar \& Ramos, 2019), muestra campos y problemáticas heterogéneas, así como diferentes ritmos y procesos, particulares de cada uno de los grupos con los cuales se trabajó. Aunque la pregunta: ¿cómo abarcar, describir y analizar la riqueza de tanta diversidad? no era nueva, volvió a aparecer con más fuerza, demandando nuestra atención.
Como menciona el Dr. Miguel León Portilla, en el libro "Toltecáyotl":

Lo más elevado de las que se nombran "instituciones" de un pueblo, las creaciones que dan apoyo a la estructuración de una cultura, todo eso y probablemente también otras realidades, se incluían en el significado de toltecáyotl... nuestros temas son el pensamiento mítico, la idea de número, tiempo y espacio entre los nahuas, sus ideales en la educación, literatura, organización social, comercio, minería y metalurgia... (León Portilla, 2013:7).

La exuberancia del universo siempre ha estado ahí. Por tanto, el estudio de los procesos de cambio de las culturas indígenas 0 ancestrales, pueden ser contemplados como objetos complejos, variados y de infinitas dimensiones. No fue sino gracias a Lotman y la Escuela de Tartu que aprendimos a ver a la cultura, en un primer momento, como un conjunto de sistemas de símbolos, y en un segundo momento, como un organismo constituido por distintos textos y lenguajes, cuya interconexión se da a lo largo y ancho de un espacio semiótico extenso. De esta manera, Lotman (2000) concibe la cultura como una totalidad heterogénea de textos, en donde bullen múltiples ritmos de desarrollo, dominantes y transitorios. Observando desde este ángulo o concepción, intentamos dar cuenta de esta previsibilidad e imprevisibilidad de los procesos sociales en las culturas originarias o ancestrales:

Separar a los individuos del espacio de los lenguajes, los signos, y los símbolos es imposible, tanto como despojarnos de la piel. En ese espacio, el individuo humano adquiere una doble vida porque es isomorfo con toda la cultura y, al mismo tiempo, es parte de esa totalidad. El primer aspecto de la existencia individual subraya su semejanza (de él o ella) a la totalidad de su cultura y conduce al rechazo de los indicadores espaciales cualitativos como insignificantes. ...sin embargo, el individuo, existe simultáneamente de forma contraria en un mundo cuantitativo. ...ambos aspectos de los que discutimos no son solo inseparables uno de otro, sino que son esenciales uno a otro (Lotman, 2013: 221),

Y como bien lo demuestran algunos trabajos en México:

La esencia pluricultural de México y la diversidad lingüística que de ella emana han provocado a lo largo de la historia grandes paradojas plasmadas en políticas lingüísticas y educación unilaterales -sean de incorporación, de integración o de autodeterminación-, contradictorias e inconsistentes todas ellas, que han conducido a un final, al parecer, irremisible: por un lado, el deslazamiento de las lenguas originarias por el español, lengua de prestigio y dominante; por otro lado, la fractura y pérdida de identidad de sus hablantes. (Barriga, 2018:39). 
En el diálogo intercultural, entender al otro se convierte, en palabras de Morin, en un acto individual de religación con el prójimo, religación con una comunidad, religación con una sociedad y, en el límite, religación con la especie humana (Morin, 2006:24). La relación de un ser humano con otro ser humano se convierte en un acto ético que obliga a establecer consonancias y disonancias, creando y estableciendo diálogos de ideas, de pensamientos y de procesos vivos. Por ello, un rasgo importante en este mar de relaciones de mundos internos y externos, es que la 'vida interna' se negocia ética y retóricamente, pues es "una práctica social en la que el discurso, el pensamiento y el sentimiento están entrelazados, con las circunstancias que los rodean, en un 'sistema dinámico de sentido"' (Shotter, 2001:73). En el mundo real, siempre estamos confrontados con la presencia del otro (otra persona, otra estructura, otro mundo, otro sistema), porque ese otro invade la vida cotidiana de uno mismo. La intrusión de lo no-sistémico dentro de lo sistémico, como menciona Lotman, constituye una de las fuentes de transformación más importantes de un modelo estático en un modelo dinámico de interacción en cualquier cultura. Son en estos espacios dinámicos de interacción en donde se pueden observar los mecanismos fundamentales de evolución que ponen en juego las culturas: el cambio y desplazamiento constante de 'uno mismo' en 'el otro', y 'del otro' en 'uno mismo': el diálogo perpetuo de las diferentes lenguas (Lotman, 2013:54-63), de las diferentes formas de estar en el mundo.

Como revela el siglo veintiuno, se ha vuelto más y más evidente que el mayor problema de nuestro tiempo energía, ambiente, cambio climático, seguridad alimenticia, seguridad financiera- no se puede entender de manera aislada. Son problemas sistémicos, lo que significa que todos ellos están interconectados y son interdependientes. A la larga, estos problemas deben verse justo como facetas diferentes de una misma crisis, la cual es, en gran parte, una crisis de percepción. Se deriva del hecho de que la mayoría de las personas en nuestra sociedad moderna, y en especial en nuestras grandes instituciones sociales, se suscriben a conceptos de visiones del mundo obsoletas, una percepción de la realidad inadecuada para tratar con un mundo sobrepoblado e interconectado globalmente (Capra \& Luisi, 2018: XI).

\section{Consideraciones teórico-metodológicas a partir de la Propuesta}

Un principio general, desde el que parte la investigación en ciencia, es de generar o producir nuevo conocimiento. Por lo que no podemos pasar por alto que,

...cada sociedad, con su episteme, produce un tipo de sujeto. Y lo hace en la historia... Cada época tiene su a priori histórico. No existe una sola manera de subjetivación; cada época tiene la suya (Beuchot, 2004:55).
Sujetos y cultura aparecen no sólo en la reflexión del mundo y de sí mismos, sino también en las prácticas y estrategias empleadas a lo largo del tiempo por ellos mismos. Existen trabajos acuñados y forjados desde una perspectiva ecológica, cuyos señalamientos y reflexiones metodológicas en Europa (Integrative Landscape Project), proveen ideas valiosas a considerar, de acuerdo con los rasgos de la propuesta que se ha venido planteando. Tress, Tress \& Fry (2005) muestran, en varios artículos, que la falta de comprensión de los conceptos de investigación por diferentes organizaciones o instituciones interesadas en una problemática, ha tenido efectos negativos en el éxito de los diferentes proyectos planteados desde una perspectiva ecológica. Sin embargo, reconocen que la producción de nuevos conocimientos -también en forma de nuevas teorías y métodos- es una característica de la investigación integradora.

Por integración entendemos que las diferentes culturas del conocimiento están unidas y su conocimiento fusionado al responder una pregunta de investigación. [...] Por proyectos de investigación integradora, se entiende que la pregunta de investigación se define conjuntamente y la respuesta a la investigación deriva de una integración de conocimientos disciplinarios. ...la integración debe conducir al desarrollo de nuevos métodos y teorías comunes y finalmente a nuevos conocimientos (Tress, Tress \& Fry, 2005:18).

Figura 1. Investigación integradora

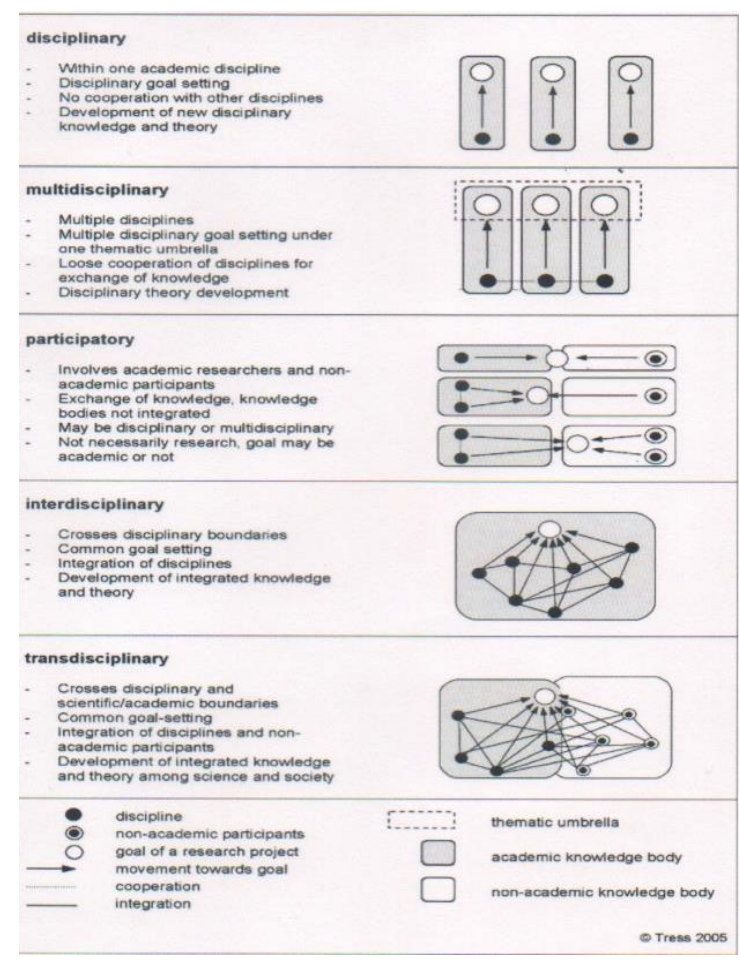

Fuente: Tress, Tress \& Fry, 2005:16 
Este cuadro muestra de manera esquemática, a través de la dirección de las flechas, el punto de partida desde el cual se vislumbra y se tiene una perspectiva de la meta y de la problemática observada. Proceder de manera lineal o multilineal será fundamental en el desarrollo de los tejidos, procesos y estrategias que se tomen en cuenta desde el inicio mismo del trabajo, capaz de integrar o no integrar conocimientos o saberes producidos, conservados y actualizados por las diferentes comunidades de conocimiento (académicas y no académicas). El cuadro ilustra, entonces en perspectiva, tanto el punto de partida como el punto de llegada del proceso de producción de conocimiento específico y genérico en la investigación entendida de manera integradora.

Como menciona el profesor Alfredo López Austin, con un énfasis particular, en el libro: "El modelo en la ciencia y en la cultura", existe una pregunta fundamental que es imposible olvidar:

¿Qué utilidad puede tener para la ciencia o la filosofía el conocimiento de concepciones paralelas, frutos de diferentes construcciones de pensamiento o de culturas del pasado distante? Ante todo existe, la imperiosa necesidad de comprender mejor la realidad propia, y esa meta se alcanza, al menos parcialmente, cuando se la compara con realidades ajenas (López Austin, 2005:6869).

Schutz dice que, aunque es posible hablar de subuniversos múltiples de la realidad, es preferible hablar de ámbitos finitos de sentido "porque lo que constituye la realidad es el sentido de nuestras experiencias, y no la estructura ontológica de los objetos" (Schutz, 1995: 215). Visto así, la realidad no es sino un conjunto de tejidos de sentido conformada por la experiencia de los sujetos que la constituyen. Universos de elementos que se desenvuelven o plasman dentro de un todo con sentido, como lo desarrollan Capra y Luisi: esa totalidad en donde las redes de todos los sistemas vivos, patrones básicos de organización, redes que se autogeneran en cada interacción con el ambiente, cambios estructurales dentro del mismo sistema, etc., se encuentran en una dinámica constante (Capra \& Luisi, 2018:144-145).

Retomando a Schutz, debemos partir de reconocer que las comunidades sociales generan, constantemente, subuniversos múltiples de realidad. Esto es, ámbitos finitos de sentido, los cuales se atraviesan y comparten en la vida diaria y cotidiana todos los sujetos sociales. Por tanto, la idea no es excluir esos ámbitos de sentido, sino más bien comprenderlos e integrarlos.

Johannes Neurath menciona que el trabajo de campo que realiza en las regiones del Gran Nayar y el Suroeste de los Estados Unidos,

...debe plantearse en términos de un sistema de transformaciones. [Porque] para la comprensión cabal de procesos culturales macro-regionales no nos sirve el particularismo a ultranza, pero tampoco las propuestas que pasan por alto las peculiaridades culturales... Lo que queremos argumentar es que sólo el análisis conjunto de semejanzas y divergencias permite alcanzar una visión, donde los datos de un grupo arrojan luz sobre los demás (Neurath, 2008:335).

José Alejos, trabajando en el sureste de México, menciona, en el libro: "Dialogismo y Semiótica de Cuentos Míticos Mayas":

En el fondo, ha sido una búsqueda de conocimientos culturales mediante procedimientos científicos, de teorías y métodos, con los cuales efectuar análisis e interpretaciones de la cultura a través de la narrativa oral tradicional... [para mostrar], a fin de cuentas, una creación estética verbal, reconocida en su momento por la comunidad que la generó, y por el mundo contemporáneo como creación artística (Alejos, 2018:12).

El propósito de los rasgos que toda propuesta debería considerar, al desarrollar trabajo con las culturas ancestrales, es entonces, alcanzar o atisbar una región de entendimiento común que permita integrar y compartir experiencias de esos tejidos complejos, en los que nos encontrarnos unos a otros a través del diálogo. Sabemos que los textos de las culturas son productos de una pluralidad de códigos en donde hay distintos participantes que generan sus propios mecanismos de traducción. Esos textos que explican y construyen el mundo son imposibles de estudiar fuera de la historia. El trabajo transdisciplinario a que se refieren Tress, Tress \& Fry (2005), se ilustra con otra imagen en donde se puede observar la necesidad de integrar saberes procedentes de distintos sujetos sociales.

Figura 2. La integración de saberes

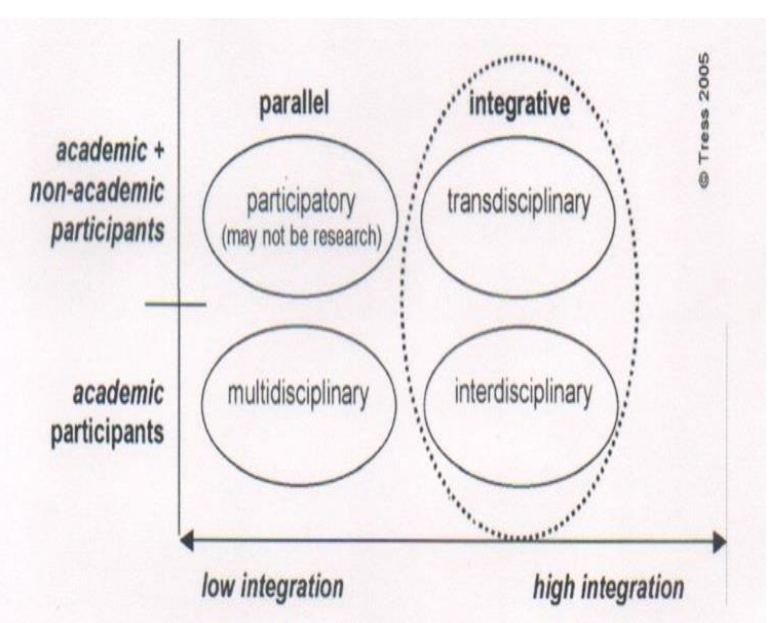

Fuente: Tress, Tress \& Fry 2005:17

Tal vez resulta un poco ambicioso, pero la idea es lograr el máximo nivel de integración de los distintos actores que se encuentran inmersos en las problemáticas 
sociales. Mencionemos brevemente otros casos con el objeto de ejemplificar el punto. En el caso del pueblo tojolabal, dice Lenkersdorf: "La integración en la llamada cultura nacional monolítica no ha funcionado porque ésta no existe, de hecho es pluralista, y como tal, es tarea de todos nosotros trabajar para esta pluralidad" (Lenkersdorf 2005:22). Martin Tonalmeyotl, quien coordinó una antología de textos poéticos, en su libro: "Xochitjajtoli. Poesía contemporánea en lenguas originarias de México", llevó a cabo su trabajo con individuos provenientes de distintas comunidades o pueblos originarios, siendo todos ellos hablantes de un idioma, que como lengua materna, no aprendió el español en primer lugar. Unas líneas, en el prólogo de dicho libro, reflejan parcialmente la pluralidad cultural a la que hemos estado haciendo referencia al realizar una aproximación compleja, sistémica y transdisciplinar:

En el listado de idiomas participantes en esta antología aparecen el náhuatl, totonaco, tsotsil, maya, mazahua, zoque, otomí, mixe, tlapaneco, zapoteco, mixteco, tzeltal, ch'ol, chontal de Tabasco, chinanteco, mazateco. Cada lengua representa un pueblo y cada pueblo tiene sus propias formas de manifestar las palabras desde la poesía, la filosofía, la medicina, entre otras áreas en donde la palabra es semilla y fruto a la vez (Tonalmeyotl, 2019:8).

Recordando a Carlos Montemayor, Tonameyotl valora un aspecto fundamental en la forma en que este escritor se acercó a trabajar con las diferentes comunidades indígenas:

Montemayor en una de sus notas sobre literatura en lenguas mexicanas menciona cuán importante es que los escritores puedan opinar desde un yo personal con los recursos y las características de una lengua y un pensamiento propio. Señala: 'con estos escritores tenemos la posibilidad, por vez primera, de acercarnos, a través de sus propios protagonistas, al rostro natural e íntimo, al profundo rostro de un México que aún desconocemos' (Tonalmeyotl, 2019:8).

Otro escritor y periodista mexicano, Hermann Bellinghausen, también ha hecho patente estas nuevas formas de expresividad, a lo largo de varios años de atestiguar los laberintos que recorre la gente que procede de los pueblos originarios, haciendo suyas también algunas de esas expresiones: "Sé que esas culturas, esos pueblos, esos idiomas profundos y nítidos, son los que mejor podrían decirnos ahora qué es México, qué no hemos descubierto aún de nosotros mismos" (Bellinghausen, 2018:18).

El sueño, la muerte y el tiempo son procesos culturales complejos en los que los seres humanos acuerdan, disponen y muestran diversos rostros y 'costumbres de vida'. Como indica Paul Ricoeur, "El sujeto no se conoce a sí mismo directamente, sino sólo a través de los signos depositados en su memoria y su imaginario por las grandes culturas" (Ricoeur, 1995:32). Por ello, el análisis del sentido, como nos recuerda Julieta Haidar, es un núcleo problemático que nos permite observar un tejido epistemológico y teórico-metodológico de las ciencias del lenguaje, de las ciencias sociales y de las ciencias artísticas (Haidar, 2005). De manera que, tanto de forma visible como de forma invisible, los funcionamientos del sentido pueden ser abordados a través de la producción y reproducción de las prácticas semiótico-discursivas de los seres humanos. Ya que ningún rasgo de la cultura está exento de la construcción de significados con los que convivimos todos los días, observar, la tarea de describir y analizar estas prácticas semiótico-discursivas será el elemento clave con el que podemos contar al tratar de entender, qué ha sucedido y qué sigue sucediendo en las comunidades y los pueblos originarios en este país.

Cuando escribimos acerca de las vidas, llevamos el mundo de los otros hacia nuestros textos. Creamos diferencias, oposiciones y presencias que nos permiten mantener la ilusión de que hemos capturado la experiencia real 'de la gente real'. De hecho, creamos a las personas de quienes escribimos, de la misma manera que ellas se crean a sí mismas cuando se entregan a las prácticas narrativas. Las vidas y los relatos que oímos y que estudiamos nos son dados bajo una promesa, y como respuesta y a cambio, este compartir nos permitirá escribir documentos de vida que hablen de la dignidad humana, del sufrimiento, de las esperanzas, de los sueños, de las vidas ganadas y de las vidas perdidas de la gente que estudiamos (Denzin, 1989:82).

\section{Algunas reflexiones a manera de cierre}

El trabajo en ciencias sociales, a lo largo del tiempo, se ha alimentado de contribuciones de diferente clase y calidad que apuntan, en momentos muy diversos, a realizar consideraciones sobre la forma del trabajo mismo que se está llevando a cabo. Dichos momentos o procesos llevan años. James Clifford escribió en la década de los 80 un libro titulado en español, "Itinerarios transculturales", en donde nos habla de las culturas viajeras, la disciplina, la identidad, los museos, los inmigrantes, los desplazamientos, entre otros aspectos. El libro intenta ser un espejo de lo que pasaba en las ciencias sociales a fines del siglo veinte, a partir de una premisa que él plantea para el trabajo de campo a lo largo del libro:

"Itinerarios transculturales", comienza con esta premisa de movimiento, y sostiene que los viajes y los contactos son situaciones cruciales para una modernidad que todavía no ha acabado de configurarse. El tópico general, si así se lo puede llamar, es muy vasto: una imagen de la ubicación humana, constituida tanto por el 
desplazamiento como por la inmovilidad (Clifford, 2008:12).

Como se puede ver, las metáforas y las paradojas se muestran de continuo como formando parte de una unidad mayor a tener en cuenta siempre, en este caso, la unidad de un sistema vivo desde donde es posible observar desplazamiento e inmovilidad. De alguna forma, son los rasgos o características de lo que una propuesta debería considerar ante estos desafíos, y que es de lo que hemos abordado a lo largo de este ensayo. Nos invita entonces, a intentar observar esas imágenes de lo humano teniendo en cuenta una perspectiva o un ángulo de sistemas dinámicos vivos en constante cambio. El cambio y las transformaciones siguen regularidades que no estamos acostumbrados a contemplar como formando parte de un continuo o unidad mayor.

Otro caso que nos gustaría recuperar como básico, al considerar el trabajo que se realiza con las culturas ancestrales, es la compilación que realizan Watzlawicck \& Krieg en el libro, "El ojo del observador", en la década de los 90s. Un libro, como dicen los autores, que "fue pensado como homenaje y agradecimiento a Heinz von Foster de parte de algunos de sus amigos" (Watzlawicck \& Krieg 1995:17). Todo el libro compila 15 documentos de primera clase, tomando en cuenta el prólogo y la introducción. Citemos sólo un pequeño fragmento del último de los artículos escrito por Varela y Depuy,

Nuestra forma de honrarlo [haciendo referencia a von Foster] consistirá en dedicarle este ensayo, que puede considerarse como una variación de un tema que ha estado en el centro de los intereses de von Foster: el papel de las circularidades como mecanismos creativos para una variedad de fenómenos, desde los vivientes a los sociales (Varela \& Depuy, 1995:232).

El libro en su conjunto intenta hacer que los lectores perciban un matiz fundamental en toda investigación y en todo acercamiento a la realidad, la 'belleza que nace en los ojos del observador' (como se menciona en el libro), para tocar y tratar de entender el mundo.

La ciencia y la filosofía [añadirá Ceruti, otro colaborador en ese mismo libro] son la sede en la que se elaboran nuevos procedimientos e imágenes de las relaciones entre lo subjetivo y lo objetivo, lo individual y lo colectivo, lo local y lo global. Son la sede de elaboración y experimentación de nuevas comunicaciones, interconexiones e hibridaciones entre la multiplicidad heterogénea de los lenguajes, de los universos locales (Ceruti, 1995:58).

Todos estos epítetos, precisamente en el contexto de la 'ciencia nueva', muestran la gama de posibilidades en las que la propuesta que hemos planteado en este ensayo, nos invita a reconsiderar en futuras investigaciones: lenguajes, mecanismos, universos, relaciones. La multidisciplinariedad, la interdisciplinariedad 0 la transdisciplinariedad, ya han sido recorridas por distintos autores durante varias décadas y ésta es una de las formas en las cuales cristaliza.

Un tercer caso, es el de Gregory Bateson. Sus trabajos e ideas fueron base de estudios y reflexiones posteriores, como las realizadas por Capra y Luigi durante años, que aquí retomamos y que conforman los cimientos conceptuales de este artículo. En el libro que lleva por título: "Pasos hacia una ecología de la mente", Bateson escribe:

La naturaleza del 'significado', la estructuración mediante patrones, la redundancia, información y conceptos análogos, depende del punto de vista donde nos situemos... en un universo más amplio, por ejemplo, el definido por el punto de vista del observador, el acto no se presenta como una 'transmisión' de información sino más bien como una diseminación de redundancia. Las actividades de $A$ y $B$ se combinaron para hacer que el universo del observador sea más predecible y más redundante (Bateson, 1991:278-279).

Como es posible atestiguar, los andamios teóricoconceptuales se enriquecen por los detalles que aparecen tanto en los universos de sentido (como son las teorías), como en los universos de vida de toda cultura. Es imposible entender a las culturas ancestrales, que han sufrido cambios y transformaciones durante cientos de años, y que poseen una 'historia larga', sin ideas o estructuras conceptuales que no perciban las complejidades de las que nos encontramos rodeados. Por tanto, lo que hacemos es una invitación al diálogo, utilizando redes de nuevos conceptos que nos permiten abrir otros horizontes, tales como: cambios estructurales, situaciones de equilibrio, auto-organización, estructuras disipativas, equilibrio dinámico, redes de patrones, emergencia, equilibrio dinámico, entre otros. Es imposible que no haya nuevos y distintos ángulos desde los cuales participar, hacer observaciones y hacer críticas. Sigamos caminando.

Esperamos que este análisis sobre las características de una propuesta de trabajo en diferentes entornos culturales sea capaz de contribuir, como una pequeña semilla, a salir de ese círculo vicioso que ha rondado a las culturas ancestrales en este país. No somos los únicos que lo hemos presenciado:

En los últimos treinta años se ha vuelto más claro de que un entendimiento pleno de estos problemas requiere nada menos que de una concepción de la vida radicalmente nueva. De hecho, tal comprensión de la vida está emergiendo ahora. A la vanguardia de la ciencia contemporánea, no vemos ya al universo como una máquina compuesta de bloques de construcción elementales. Hemos descubierto que el mundo material, en el fondo, es una red de patrones inseparables de relaciones: que el planeta como un todo es un sistema vivo que se auto-regula. La visión del cuerpo humano como una máquina y de la mente como una entidad 
separada se está remplazando por otra que no ve sólo el cerebro, sino también el sistema inmune, los tejidos corporales, e incluso a cada célula como un sistema vivo cognitivo. La evolución no se ve como una lucha por la existencia, sino más bien, como una danza cooperativa (Capra \& Luisi, 2018: XI).

\section{Referencias}

Alejos, José. (2018). Dialogismo y Semiótica de Cuentos Míticos Mayas. México: UNAM - IIF.

Bateson Gregory. (1991). Pasos hacia una ecología de la mente. Argentina: LOHLÉ - LUMEN.

Barriga, Rebeca (2018). De Babel a Pentecostés. Políticas lingüísticas y lenguas indígenas, entre historias, discursos, paradojas y testimonios. México: SEP.

Beuchot, Mauricio. (2004). Historia de la filosofía en la posmodernidad. México: Editorial Torres Asociados.

Bellinghausen, Hermann (2018). Insurrección de las palabras. México: El Colegio de San Luis/Itaca.

Bonfiglioli, Carlo; Gutiérrez, Arturo \& Olavarría, María (editores) (2006). Las vías del noroeste: una macroregión indígena americana. México: IIA-UNAM.

Capra, Fritjob (2002). Las conexiones ocultas. Barcelona: Editorial Anagrama.

Capra, Fritjob \& Luisi, Pier (2018). The Systems View of Life. New York: Cambrige University Press:

Ceruti, Mauro (1995). "El mito de la omnisciencia y el ojo del observador” en: 'El ojo del observador'. España: Gedisa Editorial.

Clifford, James (2008). Itinerarios transculturales. España: Gedisa.

Denzin, Norman (1989). Interpretive Biography. USA: Sage Publications.

García, Rolando (2006). Sistemas complejos. España: Gedisa Editorial.

González Casanova, Pablo (2005). Las Nuevas Ciencias y las Humanidades. España: Anthropos.

Haidar, Julieta (2005). "El análisis del sentido: propuesta desde la complejidad y la transdisciplina" en: La arquitectura del sentido. México: CONACULTA/UNAM.

Haidar, Julieta \& Ramos, Ignacio (2019). Fronteras semióticas de la emoción. Los procesos del sentido en las culturas. México: UNAM/ENAH

INALI (2020). 68 voces - 68 corazones. Instituto Nacional de Lenguas Indígenas. https://www.inali.gob.mx/ / https://68voces.mx/

Lenkersdorf, Carlos (2005). Los hombres verdaderos. México: Siglo XXI.

León Portilla, Miguel (2013). Toltecáyotl. México: Fondo de Cultura Económica.

López Austin, Alfredo (2005). "Modelos a distancia: antiguas concepciones Nahuas" en: El Modelo en la Ciencia y en la Cultura. México: UNAM/Siglo XXI.

Lotman, Yuri (1999). Cultura y explosión. España: Gedisa Editorial.

Lotman, Yuri (2000). Universe of the Mind. A semiotic Theory of Culture. USA: Indiana University Press.

Lotman, Juri (2013). The unpredictable workings of culture. Estonia: Tallin University Press.

Menéndez, Eduardo (2009). De sujetos, saberes y estructuras. Argentina: Lugar Editorial.

Morin, Edgar (2002). El Método. El Conocimiento del Conocimiento. Madrid: Ediciones Cátedra.

Morin Edgar, Roger Emilio \& Motta Raul (2006). Educar en la era planetaria. Barcelona: Gedisa.

Moscovici, Serge (2011). "El discurso de lo cotidiano y el sentido común: Prólogo" en: 'El discurso de lo cotidiano y el sentido común'. España: Anthropos.
Najmanovich, Denise (2012). Educar en tiempos agitados: Crisis, Cambio y Complejidad. Revista IRICE, 24(24), pp. 13-25. Recuperado a partir de https://ojs.rosarioconicet.gov.ar/index.php/revistairice/article/view/v24n24a03

Neurath, Johannes (2008). "Mitos cosmogónicos, grupos rituales e iniciación. Hacia una etnología comparada del Gran Nayar y del Suroeste de los Estados Unidos" en: 'Por los caminos del maíz', México: CNCA/FCE.

Nicolescu, Basarab (1997). Quelle université pour demain? Vers une évolution transdisciplinaire de l'université. Locarno, Suisse; Congrès internacional. http://perso.club-internet.fr/nicol/ciret/ https://basarab-nicolescu.fr/congres de locarno.php

Ortiz Álvarez, María Inés (2005). La población hablante de lenguas indígenas en México. México: UNAM - Instituto de Geografía.

Questa, Alessandro \& Neurath, Johannes (2018). "Rostros de otros mundos" en: 'Máscaras. Rostros de la alteridad'. Artes de México, Marzo, 2018, No. 128. México: Artes de México.

Ricoeur, Paul (1995). Autobiografía Intelectual. Argentina: Nueva Visión.

Schutz, Alfred (1995). El problema de la realidad social. Argentina: Amorrortu.

Shotter, Jonathan (2001). Realidades conversacionales. Buenos Aires: Amorrortu.

Tajfel, Henry \& Huici Casal, Carmen (1984). Grupos humanos y categorías sociales: Estudios de psicología social. Barcelona: Herder.

Tonalmeyotl, Martín (2019). Xochitjajtoli. Poesía contemporánea en lenguas originarias de México. México: Círculo de Poesía.

Tress Barbel, Tress Gunther \& Fry Gary (2005). "Defining concepts and the process of knowledge production in integrative research" en: Tress Barbel, Tress Gunther, Fry Gary \& Opdam Paul (eds.), 'From landscape research to landscape planning: aspects of integration, education and application', Heidelberg: Springer.

Varela, Francico \& Dupuy, Jean-Pierre (1995). "Circularidades creativas: para la comprensión de los orígenes” en: 'El ojo del observador'. España: Gedisa Editorial.

Varela, Francisco (2005). Conocer. España: Gedisa Editorial.

Watzlawick, Paul \& Krieg, Peter (1995). El ojo del observador. España: Gedisa Editorial. 